Supporting Information

\title{
Synergistic Effect of Chemical Substitution and Insertion on the Thermoelectric Performance of $\mathrm{Cu}_{26} \mathrm{~V}_{2} \mathrm{Ge}_{6} \mathrm{~S}_{32}$ Colusite
}

Yuta Shimizul, Koichiro Suekunil,2*, Hikaru Saito ${ }^{3}$, Pierric Lemoine ${ }^{4}$, Emmanuel Guilmeau $^{5}$, Bernard Raveau ${ }^{5}$, Raju Chetty ${ }^{6}$, Michihiro Ohta ${ }^{6}$, Toshiro Takabatake, Michitaka Ohtaki ${ }^{1,2}$

${ }^{1}$ Interdisciplinary Graduate School of Engineering Sciences, Kyushu University, Kasuga, Fukuoka 816-8580, Japan

${ }^{2}$ Transdisciplinary Research and Education Center for Green Technologies, Kyushu University, Kasuga, Fukuoka 816-8580, Japan

${ }^{3}$ Institute for Materials Chemistry and Engineering, Kyushu University, Kasuga, Fukuoka 816-8580, Japan

${ }^{4}$ Université de Rennes 1, CNRS, ISCR-UMR 6226, F-35000 Rennes, France

${ }^{5}$ CRISMAT, CNRS, Normandie Université, ENSICAEN, UNICAEN, 14000 Caen, France

${ }^{6}$ Global Zero Emission Research Center, National Institute of Advanced Industrial Science and Technology (AIST), Tsukuba, Ibaraki 305-8569, Japan

${ }^{7}$ Graduate School of Advanced Science and Engineering, Hiroshima University, Higashi-Hiroshima 739-8530, Japan 
Table S1 Sample density $d_{\mathrm{s}}\left[\mathrm{g} \mathrm{cm} \mathrm{cm}^{-3}\right]$, theoretical density $d_{\mathrm{t}}\left[\mathrm{g} \mathrm{cm} \mathrm{cm}^{-3}\right]$, and the ratio $d_{\mathrm{s}} / d_{\mathrm{t}}$ for the $\mathrm{Cu}_{26+\mathrm{y}} \mathrm{V}_{2} \mathrm{Ge}_{6-\mathrm{x}} \mathrm{Sb}_{\mathrm{x}} \mathrm{S}_{32}$ samples.

\begin{tabular}{llllllllllllll}
\hline & \multicolumn{3}{c}{$x=0$} & \multicolumn{3}{c}{$x=1$} & \multicolumn{3}{c}{$x=2$} & \multicolumn{3}{c}{$x=3$} \\
& $d_{\mathrm{s}}$ & $d_{\mathrm{t}}$ & $d_{\mathrm{s}} / d_{\mathrm{t}}$ & $d_{\mathrm{s}}$ & $d_{\mathrm{t}}$ & $d_{\mathrm{s}} / d_{\mathrm{t}}$ & $d_{\mathrm{s}}$ & $d_{\mathrm{t}}$ & $d_{\mathrm{s}} / d_{\mathrm{t}}$ & $d_{\mathrm{s}}$ & $d_{\mathrm{t}}$ & $d_{\mathrm{s}} / d_{\mathrm{t}}$ \\
\hline$y=0$ & 4.40 & 4.52 & 0.97 & 4.44 & 4.55 & 0.98 & 4.41 & 4.58 & 0.96 & 4.46 & 4.60 & 0.97 \\
$y=1$ & 4.43 & 4.61 & 0.96 & 4.41 & 4.63 & 0.95 & - & - & - & - & - & - \\
$y=2$ & 4.54 & 4.67 & 0.97 & 4.50 & 4.68 & 0.96 & - & - & - & - & - & - \\
$y=3$ & 4.60 & 4.73 & 0.97 & 4.57 & 4.75 & 0.96 & - & - & - & - & - & - \\
\hline
\end{tabular}
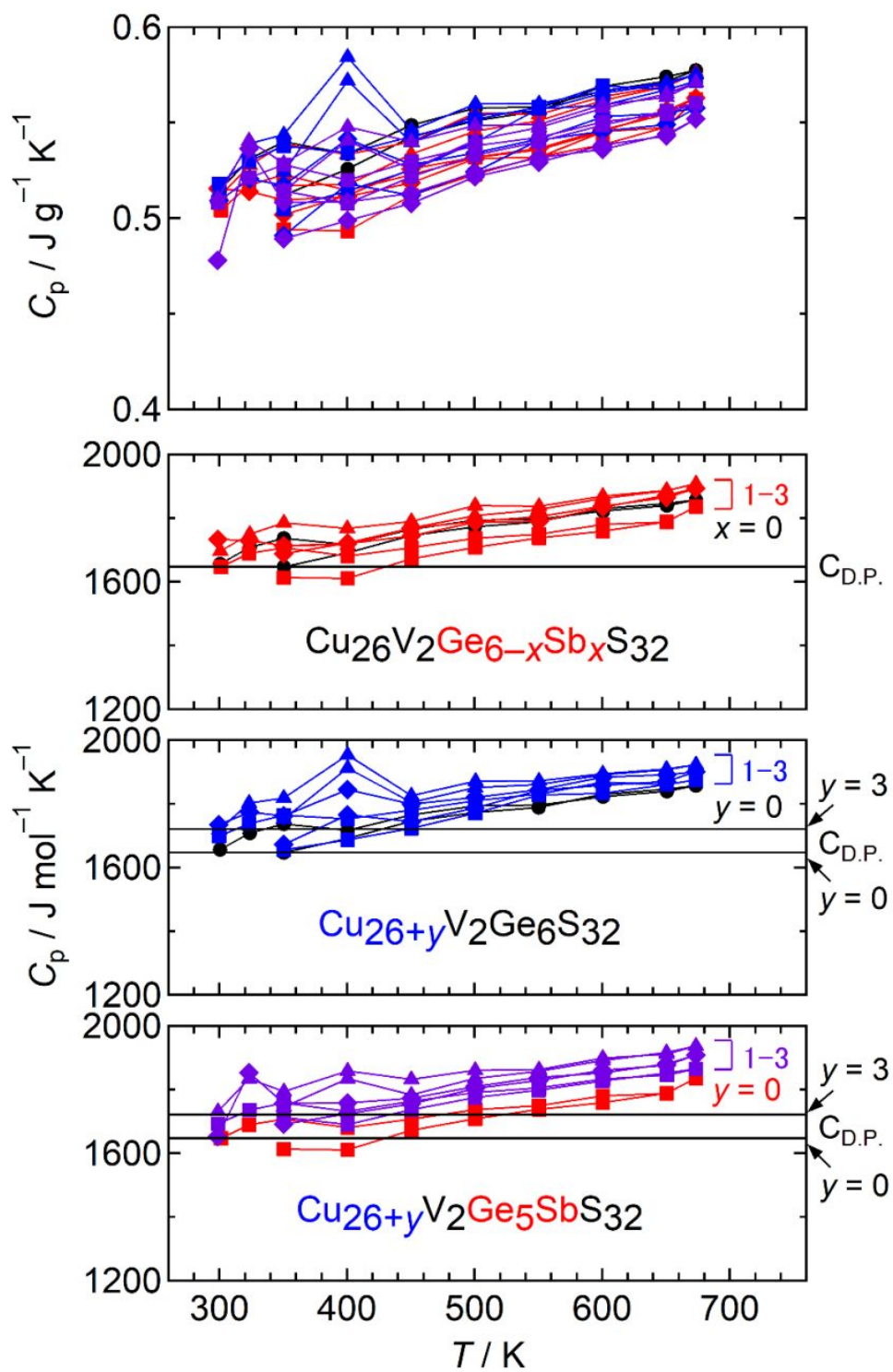

Figure S1. Specific heat $\mathrm{C}_{\mathrm{p}}$ in units of $\mathrm{J} \mathrm{g}^{-1} \mathrm{~K}^{-1}$ and $\mathrm{J} \mathrm{mol}^{-1} \mathrm{~K}^{-1}$ for $\mathrm{Cu}_{26} \mathrm{~V}_{2} \mathrm{Ge}_{6-\mathrm{x}} \mathrm{Sb}_{\mathrm{x}} \mathrm{S}_{32}, \mathrm{Cu}_{26+\mathrm{y}} \mathrm{V}_{2} \mathrm{Ge}_{6} \mathrm{~S}_{32}$, and $\mathrm{Cu}_{26+\mathrm{y}} \mathrm{V}_{2} \mathrm{Ge}_{6-\mathrm{x}} \mathrm{Sb}_{\mathrm{x}} \mathrm{S}_{32}(\mathrm{x}=1)$ samples. 

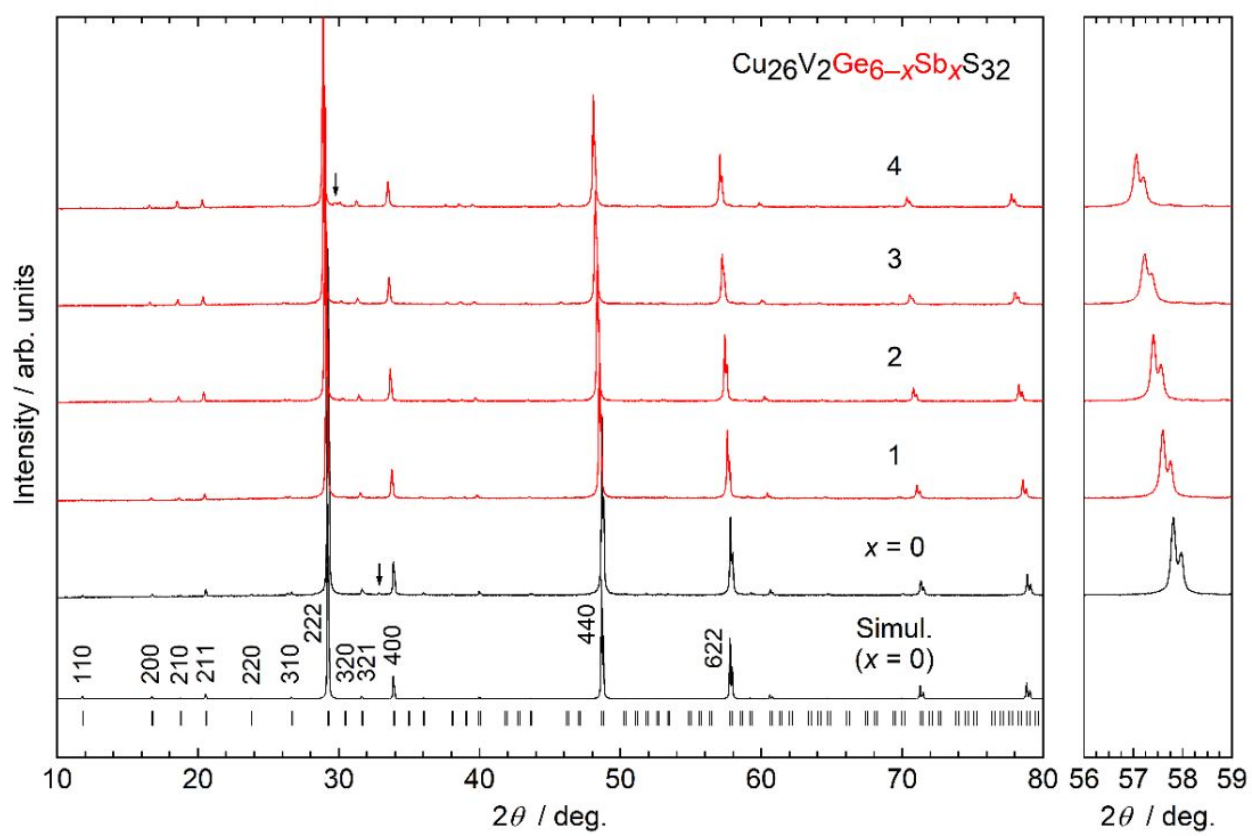

Figure S2. Powder X-ray diffraction patterns for $\mathrm{Cu}_{26} \mathrm{~V}_{2} \mathrm{Ge}_{6-x} \mathrm{Sb}_{x} \mathrm{~S}_{32}$ samples. Arrows for $x=0$ and $x=4$ denote peaks from $\mathrm{Cu}_{2} \mathrm{~S}$ and an unidentified phase, respectively. The simulated pattern based on the colusite $\left(\mathrm{Cu}_{26} \mathrm{~V}_{2} \mathrm{Ge}_{6} \mathrm{~S}_{32}\right)$ structure is shown at the bottom. The right panel shows expanded views of 622 peaks.

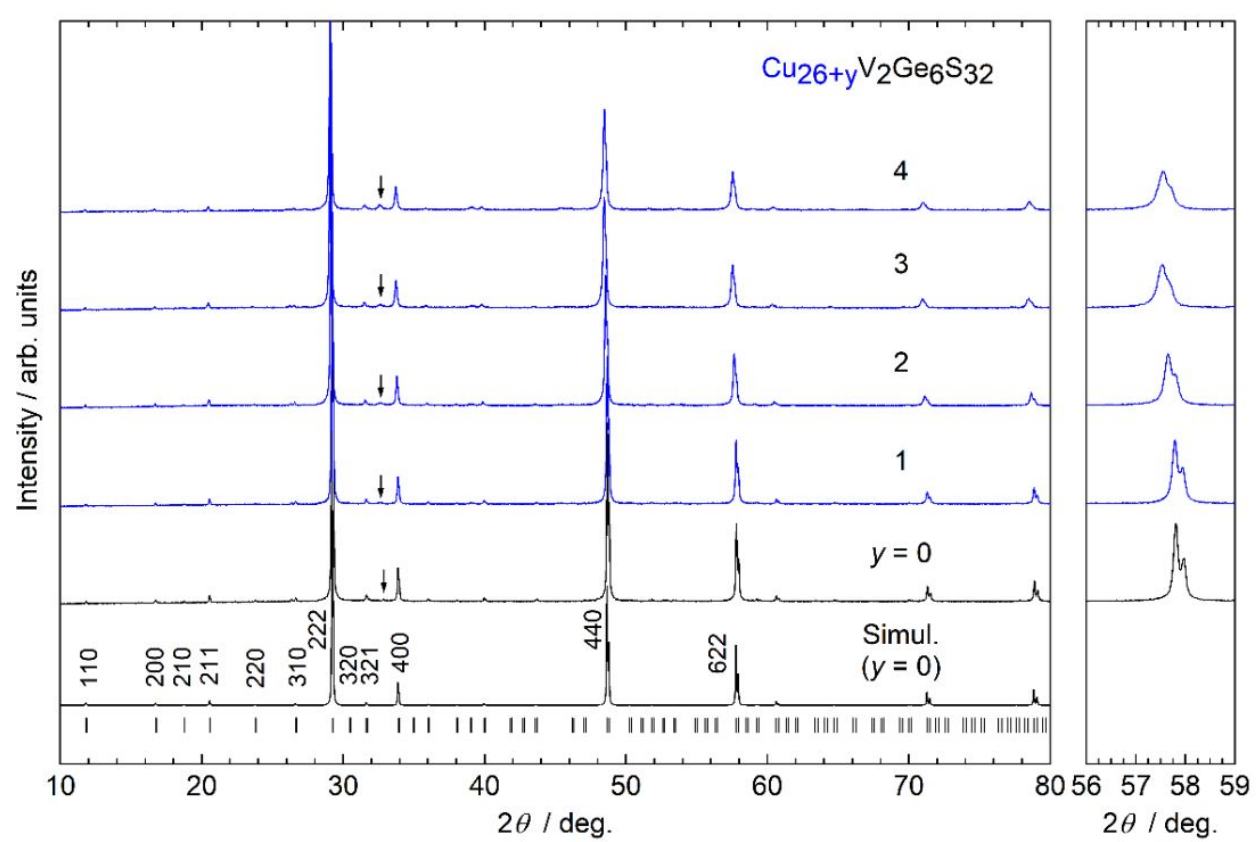

Figure S3. Powder X-ray diffraction patterns for $\mathrm{Cu}_{26+y} \mathrm{~V}_{2} \mathrm{Ge}_{6} \mathrm{~S}_{32}$ samples. Arrows denote peaks from $\mathrm{Cu}_{2} \mathrm{~S}$. The simulated pattern based on the colusite $\left(\mathrm{Cu}_{26} \mathrm{~V}_{2} \mathrm{Ge}_{6} \mathrm{~S}_{32}\right)$ structure is shown at the bottom. The right panel shows expanded views of 622 peaks. 


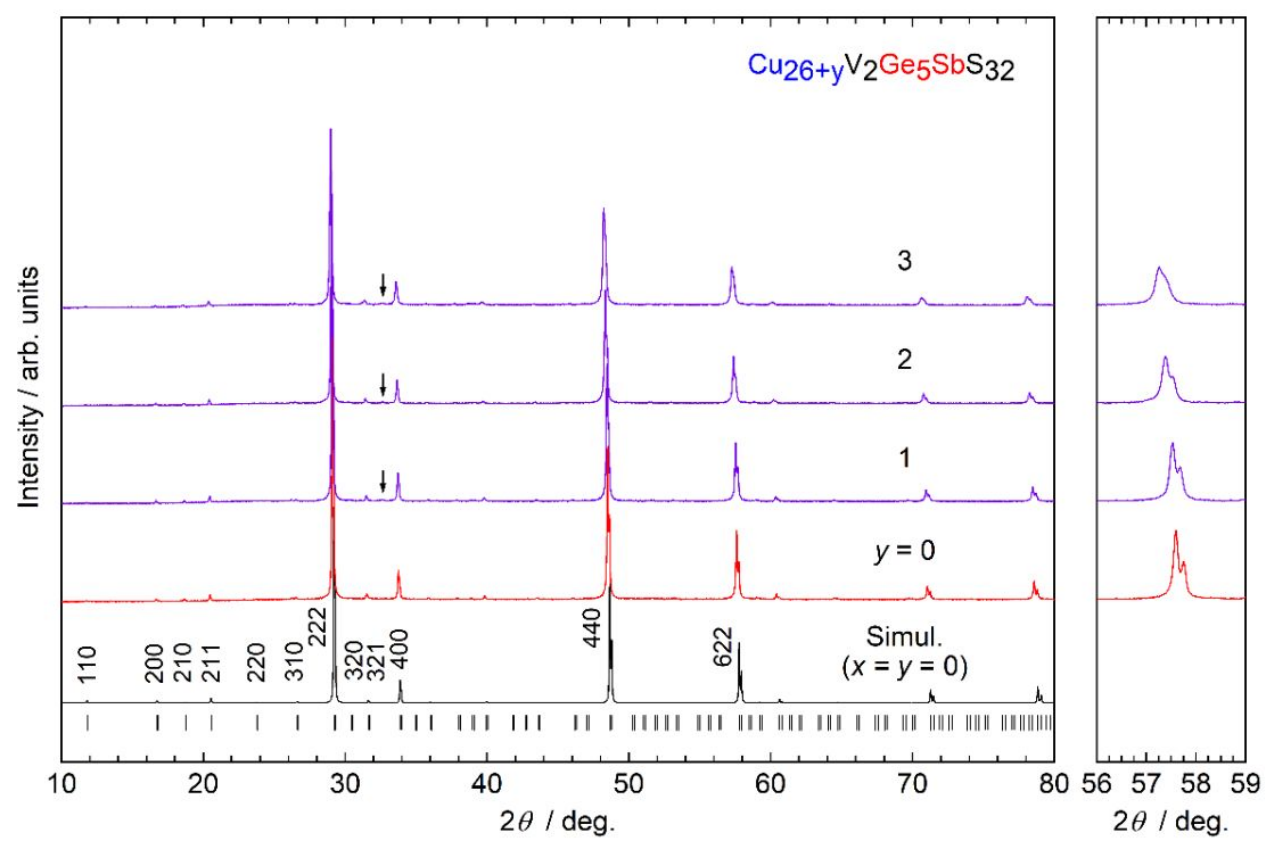

Figure S4. Powder X-ray diffraction patterns for $\mathrm{Cu}_{26+y} \mathrm{~V}_{2} \mathrm{Ge}_{6-x} \mathrm{Sb}_{x} \mathrm{~S}_{32}$ with $x=1$ samples. Arrows denote peaks from $\mathrm{Cu}_{2} \mathrm{~S}$. The simulated pattern based on the colusite $\left(\mathrm{Cu}_{26} \mathrm{~V}_{2} \mathrm{Ge}_{6} \mathrm{~S}_{32}\right)$ structure is shown at the bottom. The right panel shows expanded views of 622 peaks.

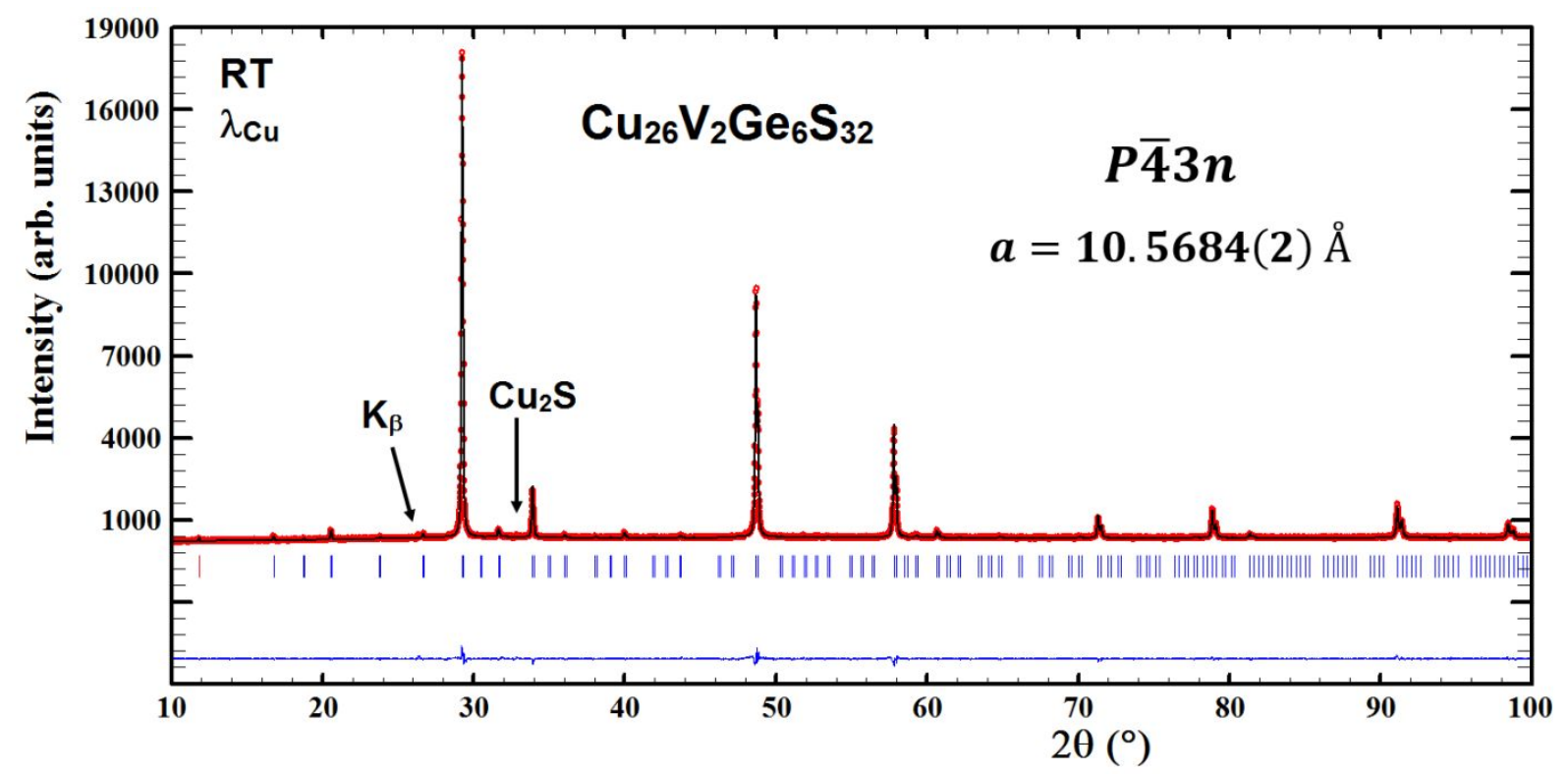

Figure S5. Rietveld refinement of power X-ray diffraction patterns for $\mathrm{Cu}_{26} \mathrm{~V}_{2} \mathrm{Ge}_{6} \mathrm{~S}_{32}$. 


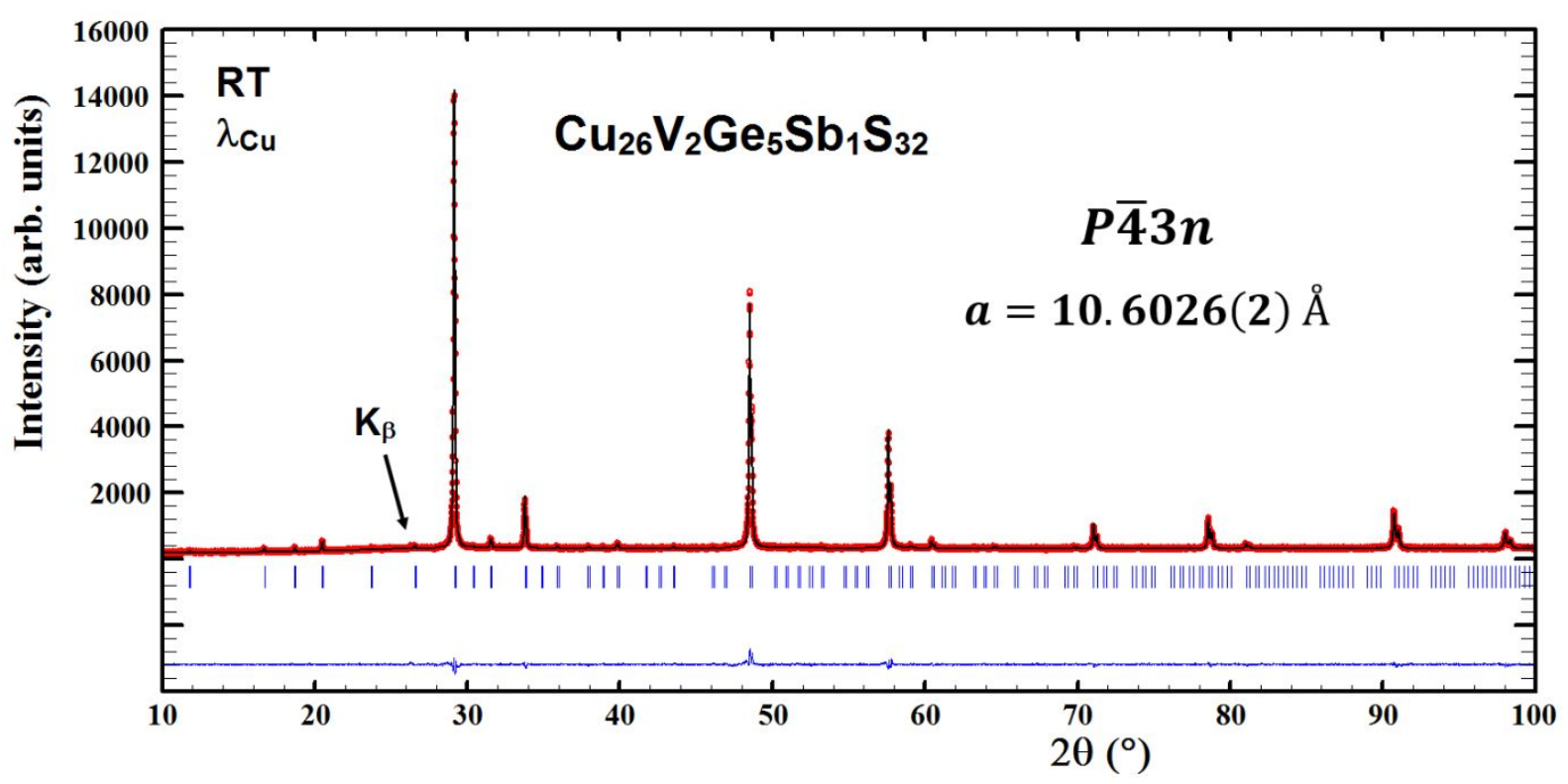

Figure S6. Rietveld refinement of power X-ray diffraction patterns for $\mathrm{Cu}_{26} \mathrm{~V}_{2} \mathrm{Ge}_{6-x} \mathrm{Sb}_{x} \mathrm{~S}_{32}$ with $x=1$.

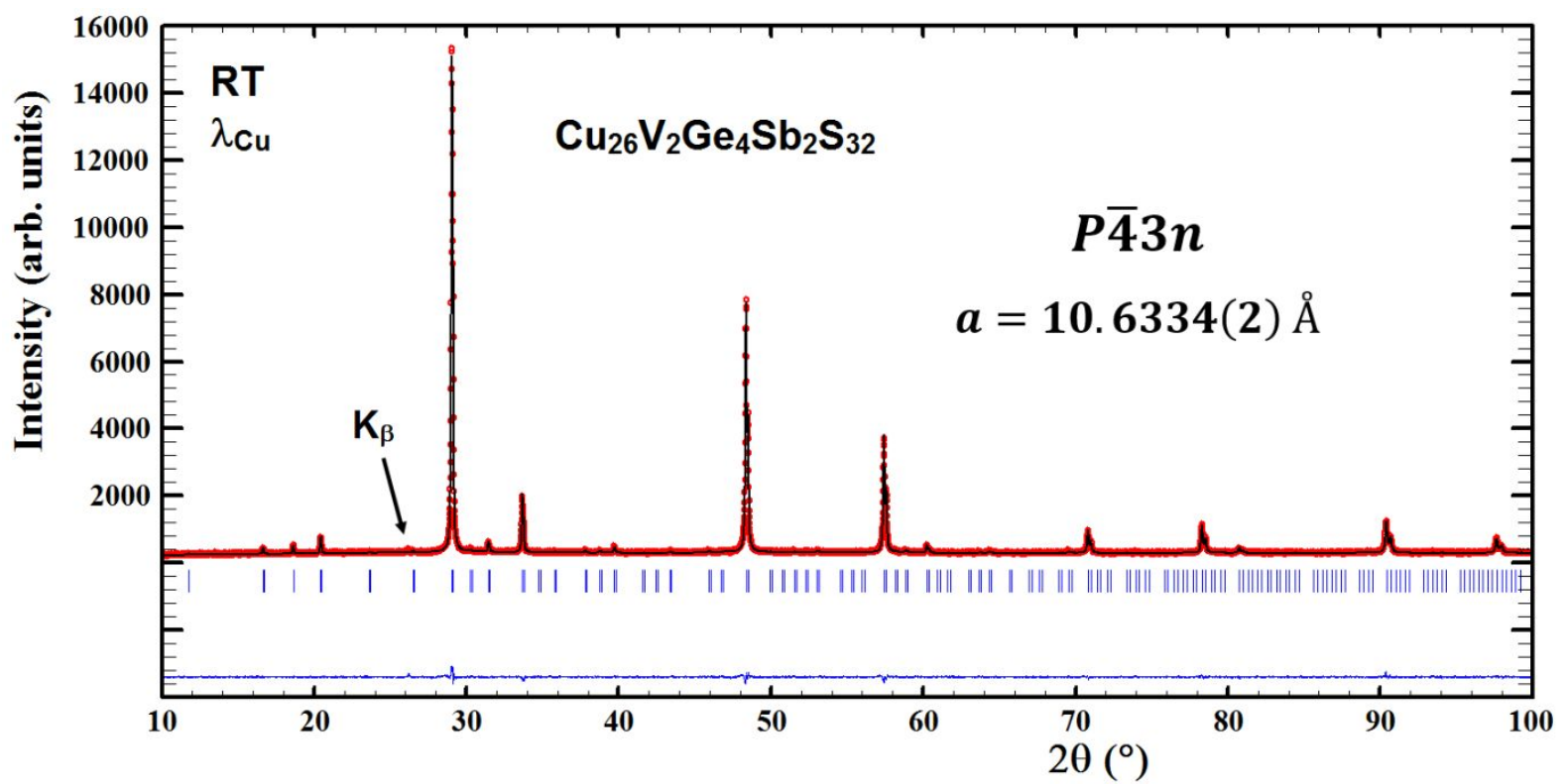

Figure S7. Rietveld refinement of power X-ray diffraction patterns for $\mathrm{Cu}_{26} \mathrm{~V}_{2} \mathrm{Ge}_{6-x} \mathrm{Sb}_{x} \mathrm{~S}_{32}$ with $x=2$. 


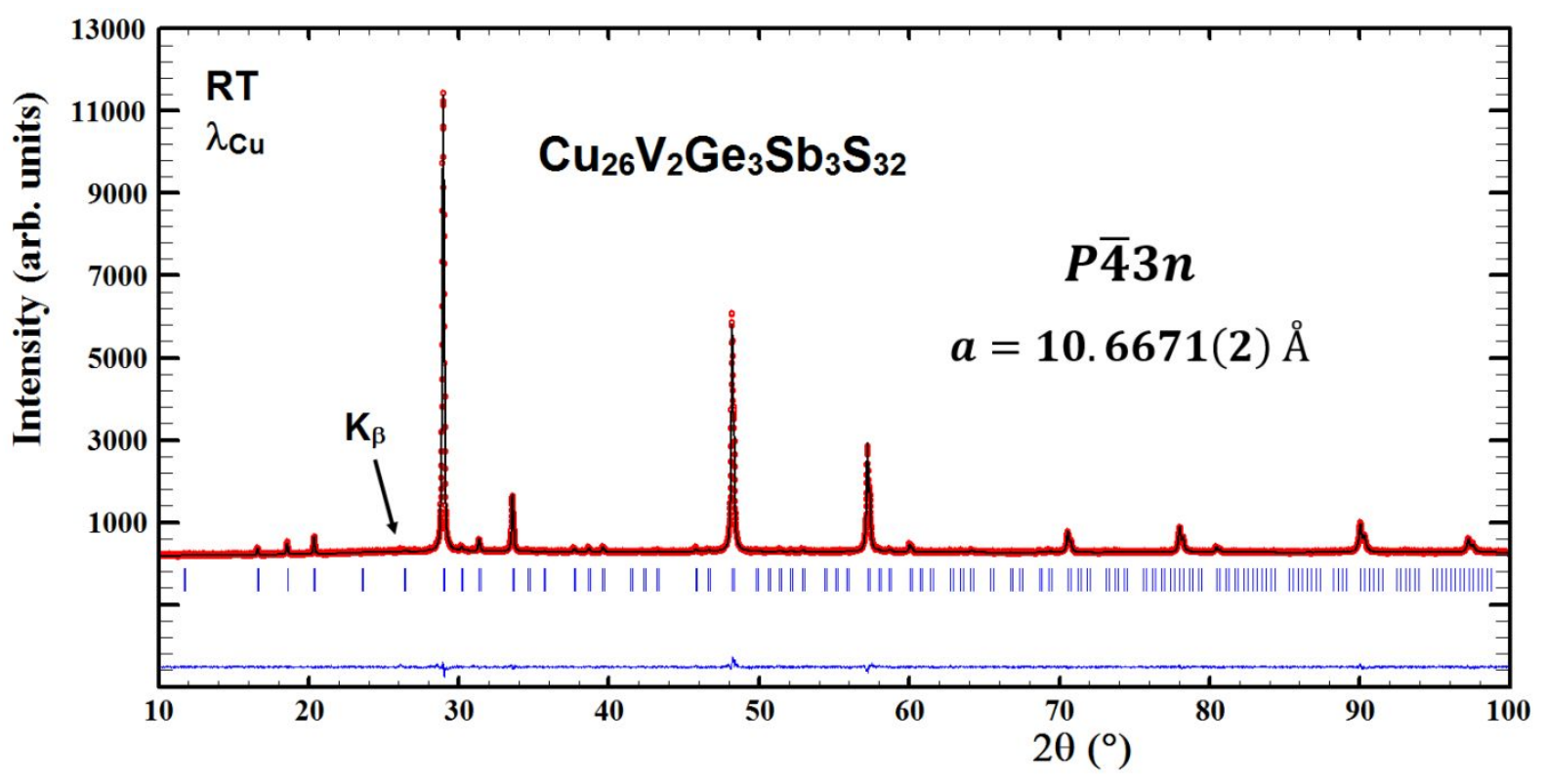

Figure S8. Rietveld refinement of power X-ray diffraction patterns for $\mathrm{Cu}_{26} \mathrm{~V}_{2} \mathrm{Ge}_{6-x} \mathrm{Sb}_{x} \mathrm{~S}_{32}$ with $x=3$.

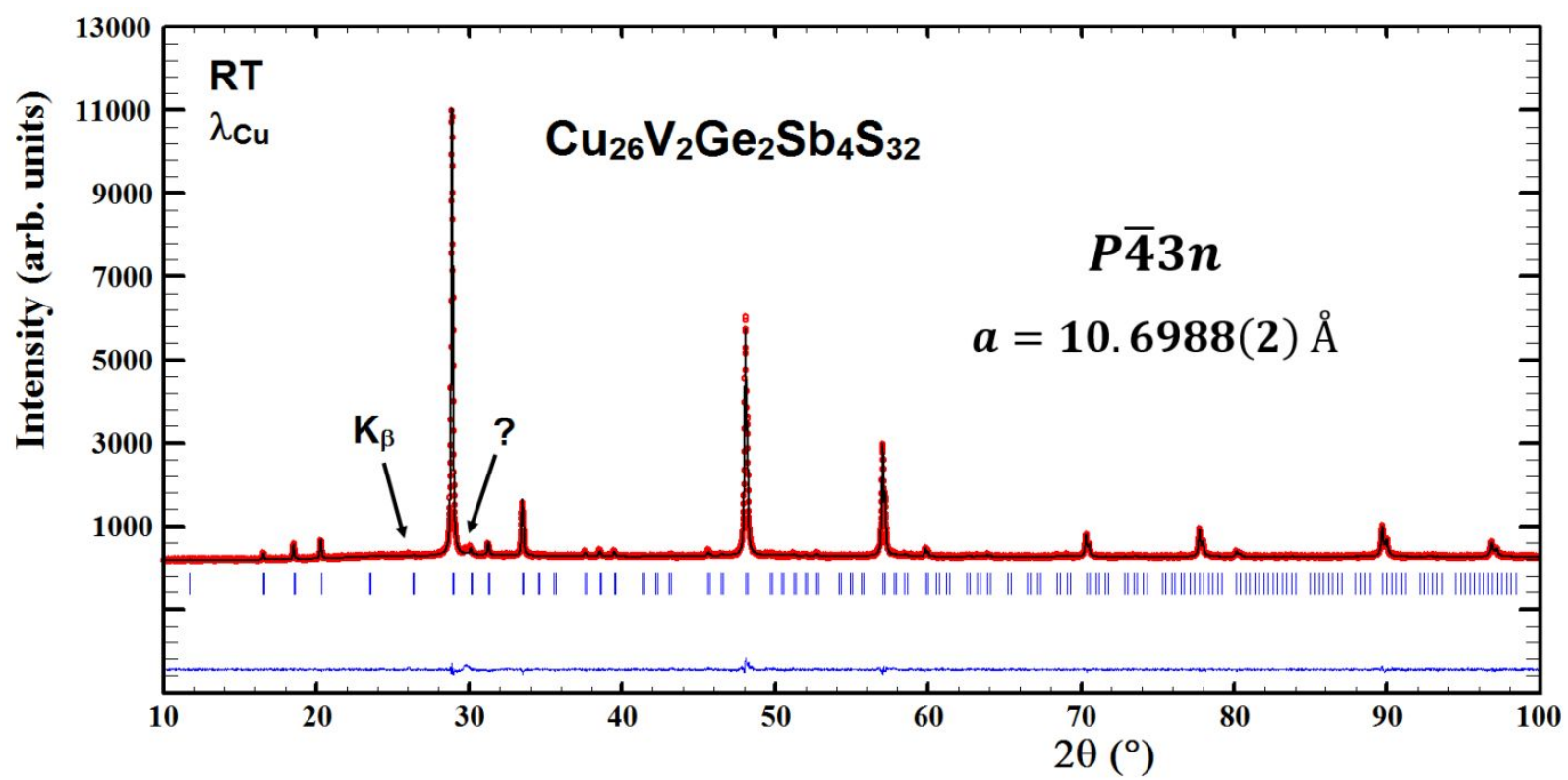

Figure S9. Rietveld refinement of power X-ray diffraction patterns for $\mathrm{Cu}_{26} \mathrm{~V}_{2} \mathrm{Ge}_{6-x} \mathrm{Sb}_{x} \mathrm{~S}_{32}$ with $x=4$. 
Table S2. Results from the Rietveld refinements of power X-ray diffraction patterns for $\mathrm{Cu}_{26} \mathrm{~V}_{2} \mathrm{Ge}_{6-x} \mathrm{Sb}_{x} \mathrm{~S}_{32}$ samples (space group $P \overline{4} 3 n$ ).

\begin{tabular}{|c|c|c|c|c|c|}
\hline $\mathrm{Cu}_{26} \mathrm{~V}_{2} \mathrm{Ge}_{6-x} \mathrm{Sb}_{x} \mathrm{~S}_{32}$ & $x=0$ & $x=1$ & $x=2$ & $x=3$ & $x=4$ \\
\hline$a(\AA)$ & $10.5684(2)$ & $10.6026(2)$ & $10.6334(2)$ & $10.6671(2)$ & $10.6988(2)$ \\
\hline SOF_Ge $(6 c)$ & 1.00 & $0.87(2)$ & $0.69(2)$ & $0.50(2)$ & $0.38(2)$ \\
\hline SOF_Sb $(6 c)$ & - & $0.13(2)$ & $0.31(2)$ & $0.50(2)$ & $0.62(2)$ \\
\hline$x_{-} \mathrm{Cu}(8 e)$ & $0.248(1)$ & $0.248(1)$ & $0.247(1)$ & $0.245(1)$ & $0.246(1)$ \\
\hline$x_{-} \mathrm{Cu}(12 f)$ & $0.260(1)$ & $0.260(1)$ & $0.257(1)$ & $0.257(1)$ & $0.256(1)$ \\
\hline$x \_\mathrm{S}(8 e)$ & $0.126(1)$ & $0.127(1)$ & $0.125(1)$ & $0.123(1)$ & $0.124(1)$ \\
\hline$x \_\mathrm{S}(24 i)$ & $0.371(1)$ & $0.371(1)$ & $0.372(1)$ & $0.375(1)$ & $0.376(1)$ \\
\hline$y_{-} \mathrm{S}(24 i)$ & $0.373(1)$ & $0.373(1)$ & $0.372(1)$ & $0.370(1)$ & $0.368(1)$ \\
\hline$z_{-} \mathrm{S}(24 i)$ & $0.121(1)$ & $0.122(1)$ & $0.122(1)$ & $0.126(1)$ & $0.128(1)$ \\
\hline$\chi^{2}$ & 0.678 & 0.641 & 0.575 & 0.560 & 0.680 \\
\hline$R_{\mathrm{wp}} ; R_{\mathrm{exp}}$ & $4.09 ; 4.96$ & $4.19 ; 5.23$ & $3.98 ; 5.25$ & $4.11 ; 5.49$ & $4.62 ; 5.60$ \\
\hline$R_{\text {Bragg }} ; R_{\mathrm{F}}$ & $2.62 ; 7.54$ & $3.05 ; 6.45$ & $2.20 ; 5.37$ & $2.72 ; 5.17$ & $3.03 ; 6.29$ \\
\hline \multicolumn{6}{|l|}{ Distances $(\AA)$} \\
\hline $\mathrm{V}(2 a)-\mathrm{S}(8 e) \times 4$ & 2.308 & 2.325 & 2.297 & 2.267 & 2.296 \\
\hline $\mathrm{V}(2 a)-\mathrm{Cu}(12 f) \times 6$ & 2.751 & 2.755 & 2.735 & 2.741 & 2.740 \\
\hline$M(6 c)-\mathrm{S}(24 i) \times 4$ & 2.254 & 2.269 & 2.291 & 2.349 & 2.383 \\
\hline $\mathrm{Cu}(6 d)-\mathrm{S}(24 i) \times 4$ & 2.276 & 2.291 & 2.281 & 2.286 & 2.286 \\
\hline $\mathrm{Cu}(8 e)-\mathrm{S}(8 e) \times 1$ & 2.237 & 2.220 & 2.245 & 2.265 & 2.255 \\
\hline $\mathrm{Cu}(8 e)-\mathrm{S}(24 i) \times 3$ & 2.286 & 2.289 & 2.303 & 2.297 & 2.293 \\
\hline $\mathrm{Cu}(12 f)-\mathrm{S}(8 e) \times 2$ & 2.359 & 2.366 & 2.346 & 2.341 & 2.348 \\
\hline $\mathrm{Cu}(12 f)-\mathrm{S}(24 i) \times 2$ & 2.287 & 2.291 & 2.313 & 2.297 & 2.300 \\
\hline $\mathrm{Cu}-\mathrm{S}$ (average) & 2.291 & 2.297 & 2.300 & 2.298 & 2.298 \\
\hline
\end{tabular}


(a)

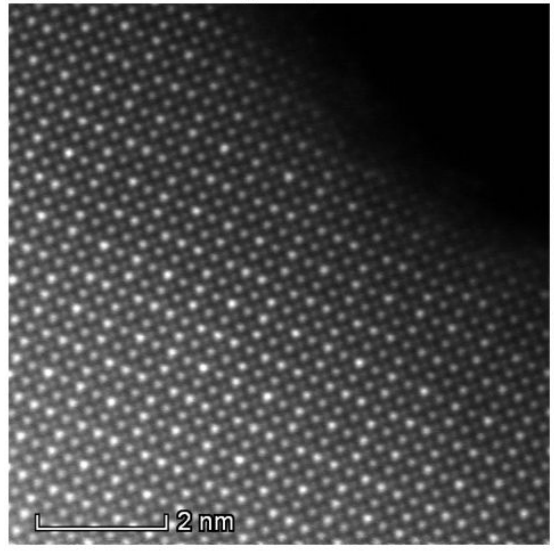

(b)

[111]

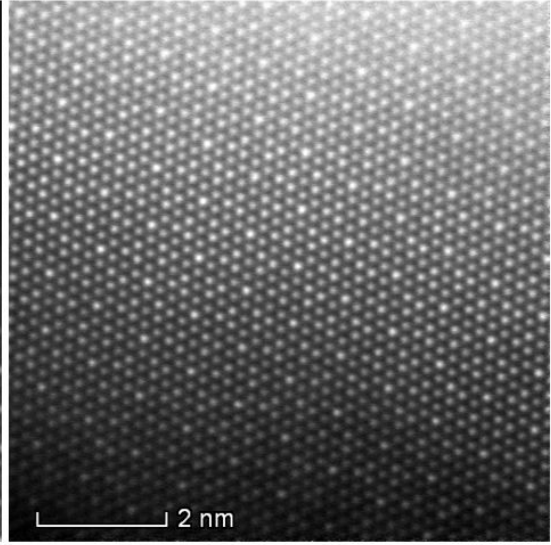

Figure S10. Annular dark-field scanning transmission electron microscopy images along the (a) 100 and (b) 111 directions for $\mathrm{Cu}_{26} \mathrm{~V}_{2} \mathrm{Ge}_{6-x} \mathrm{Sb}_{x} \mathrm{~S}_{32}(x=2)$ sample.

(a) [100]

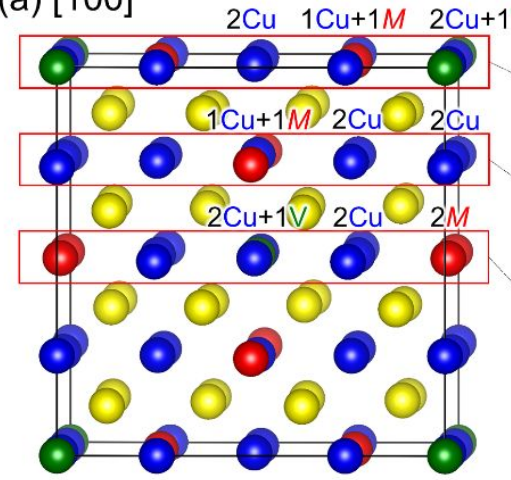

(b) [111]

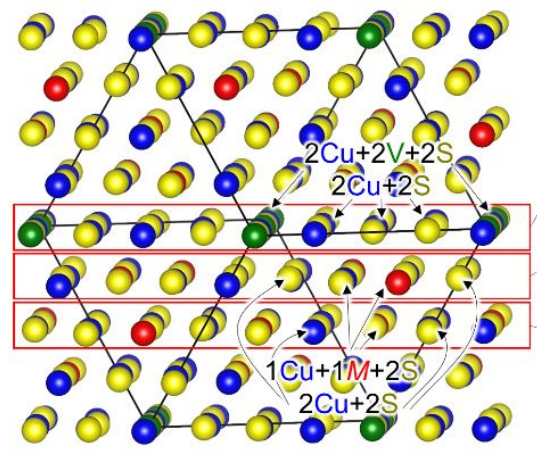

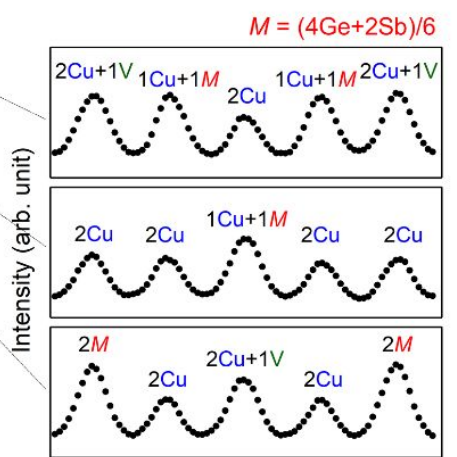

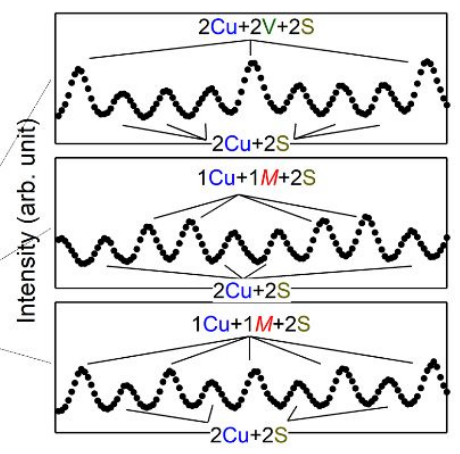

Figure S11. Line profiles of intensity for atomic columns along the (a) 100 and (b) 111 directions depicted in

Figure S10 and the corresponding view of the crystal structure. 
(a)

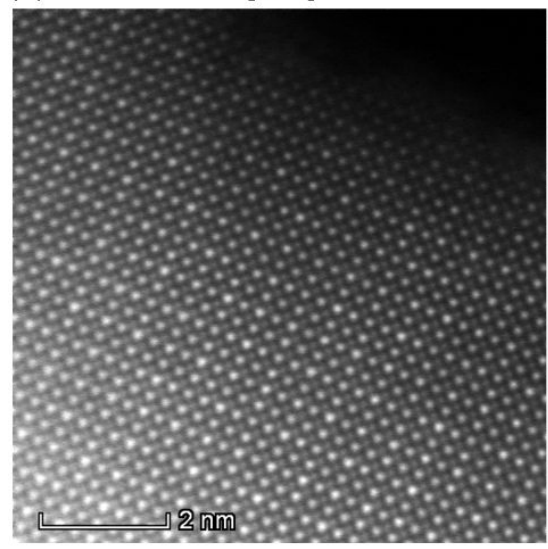

(b) [111]

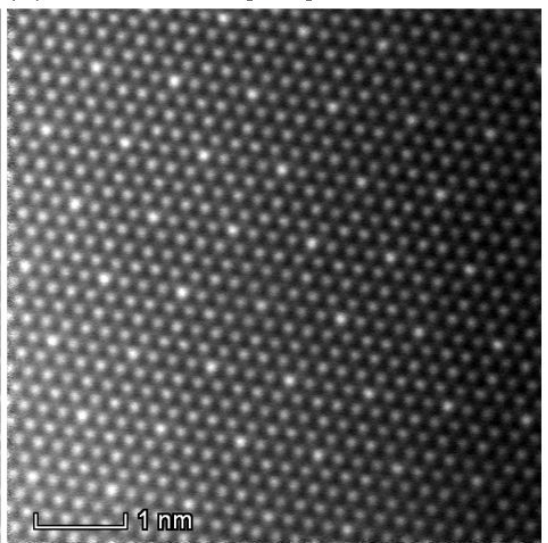

Figure S12. Annular dark-field scanning transmission electron microscopy images along the (a) 100 and (b) 111 directions for $\mathrm{Cu}_{26+y} \mathrm{~V}_{2} \mathrm{Ge}_{6} \mathrm{~S}_{32}(y=3)$ sample.

(a) [100]

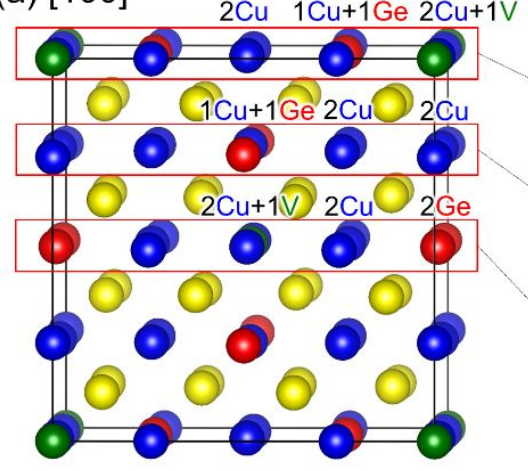

(b) [111]

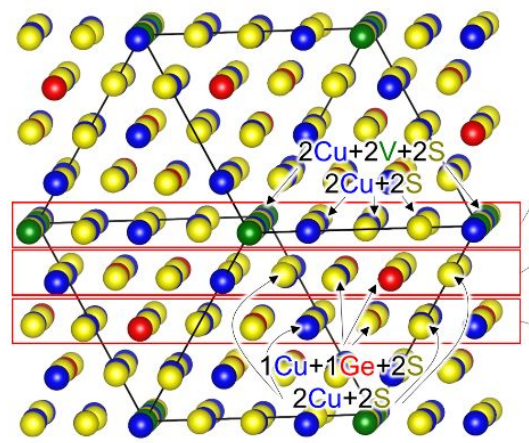

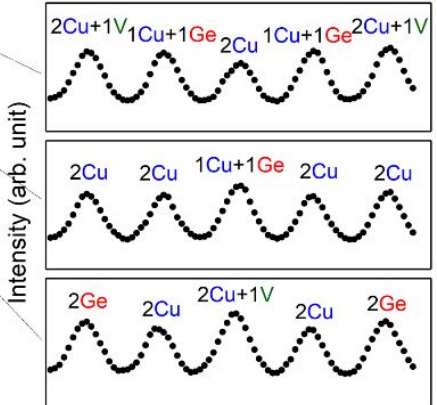

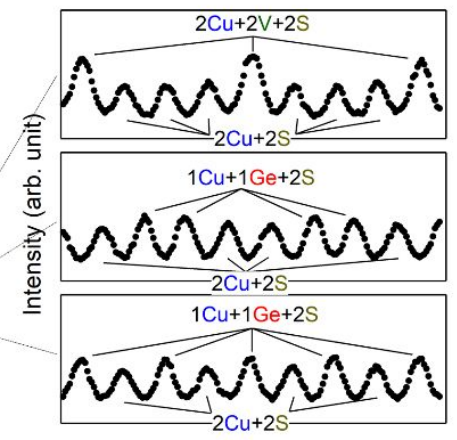

Figure S13. Line profiles of intensity for atomic columns along the (a) 100 and (b) 111 directions depicted in

Figure S12 and the corresponding view of the crystal structure. 


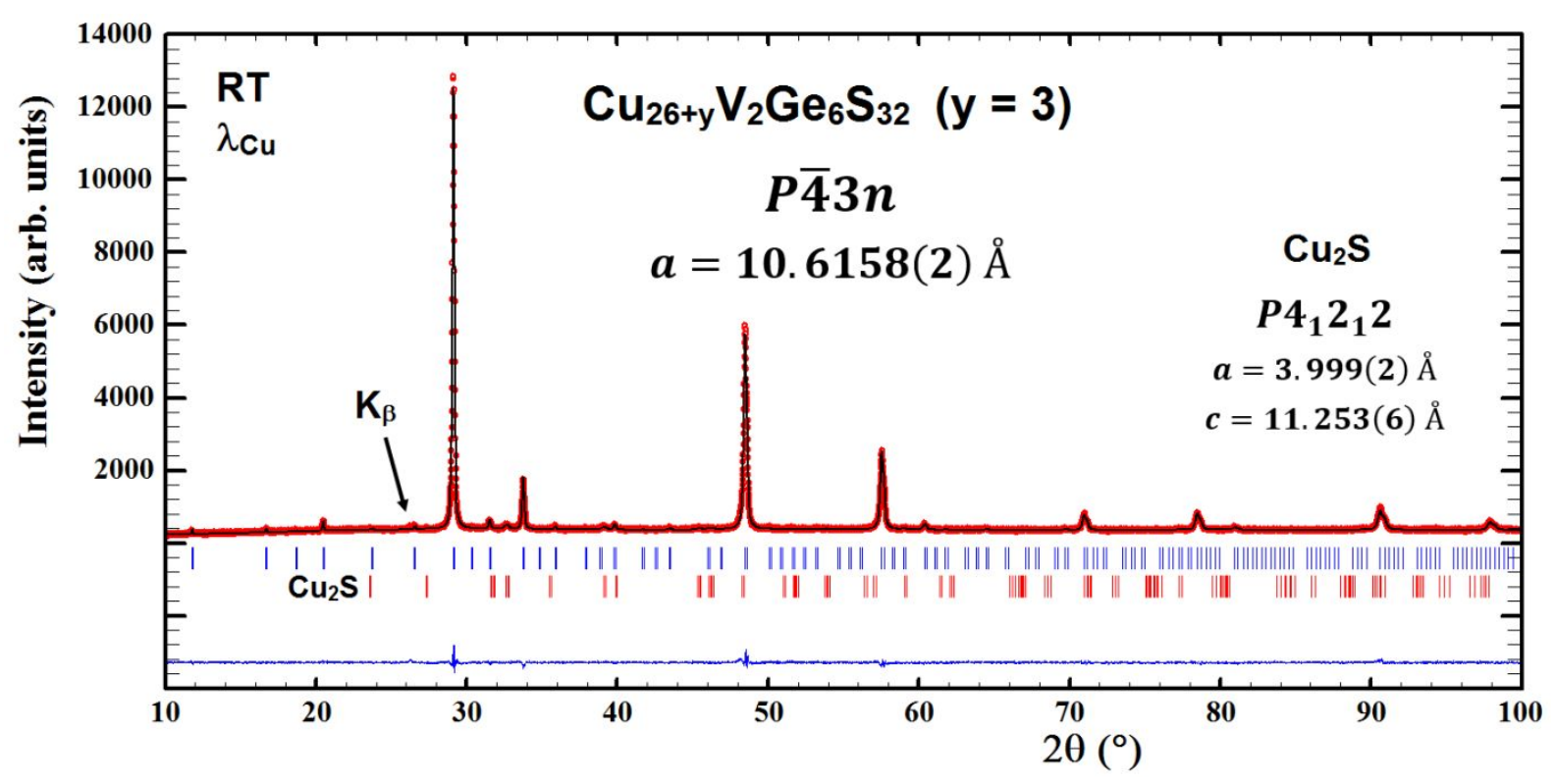

Figure S14. Rietveld refinement of power X-ray diffraction patterns for $\mathrm{Cu}_{26+y} \mathrm{~V}_{2} \mathrm{Ge}_{6} \mathrm{~S}_{32}$ with $y=3$.

Table S3. Results from Rietveld refinements of powder X-ray diffraction patterns for $y=0$ and $y=3$ samples of $\mathrm{Cu}_{26+y} \mathrm{~V}_{2} \mathrm{Ge}_{6} \mathrm{~S}_{32}$ (space group $P \overline{4} 3 n$ ).

\begin{tabular}{llll}
\hline $\mathrm{Cu}_{26+\mathrm{y}} \mathrm{V}_{2} \mathrm{Ge}_{6} \mathrm{~S}_{32}$ & $y=0$ & $y=3$ & $y=3$ \\
\hline$a(\AA)$ & $10.5684(2)$ & $10.6149(2)$ & $10.6158(2)$ \\
\hline $\mathrm{SOF} \_\mathrm{Cu}(24 i)^{*}$ & - & - & $0.042(1)$ \\
\hline$x_{-} \mathrm{Cu}(8 e)$ & $0.248(1)$ & $0.247(1)$ & $0.246(1)$ \\
$x_{-} \mathrm{Cu}(12 f)$ & $0.260(1)$ & $0.260(1)$ & $0.260(1)$ \\
$x_{-} \mathrm{S}(8 e)$ & $0.126(1)$ & $0.127(1)$ & $0.127(1)$ \\
$x_{-} \mathrm{S}(24 i)$ & $0.371(1)$ & $0.372(1)$ & $0.372(1)$ \\
$y \_\mathrm{S}(24 i)$ & $0.373(1)$ & $0.372(1)$ & $0.373(1)$ \\
$z_{-} \mathrm{S}(24 i)$ & $0.121(1)$ & $0.121(1)$ & $0.120(1)$ \\
\hline$\chi^{2}$ & 0.678 & 0.760 & 0.651 \\
$R_{\mathrm{wp}} ; R_{\text {exp }}$ & $4.09 ; 4.96$ & $4.21 ; 4.82$ & $3.88 ; 4.81$ \\
$R_{\text {Bragg }} ; R_{\mathrm{F}}$ & $2.62 ; 7.54$ & $7.21 ; 10.5$ & $4.11 ; 9.01$ \\
\hline$*$ & $\mathrm{Cu}$ atoms in interstitial position $(0.236,0.235,0.007)$
\end{tabular}




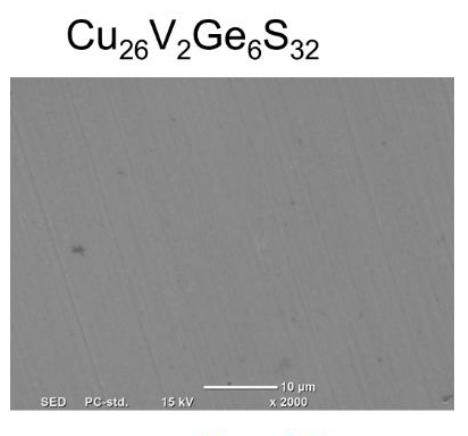

$\mathrm{Cu}_{26} \mathrm{~V}_{2} \mathrm{Ge}_{6-x} \mathrm{Sb}_{x} \mathrm{~S}_{32}$
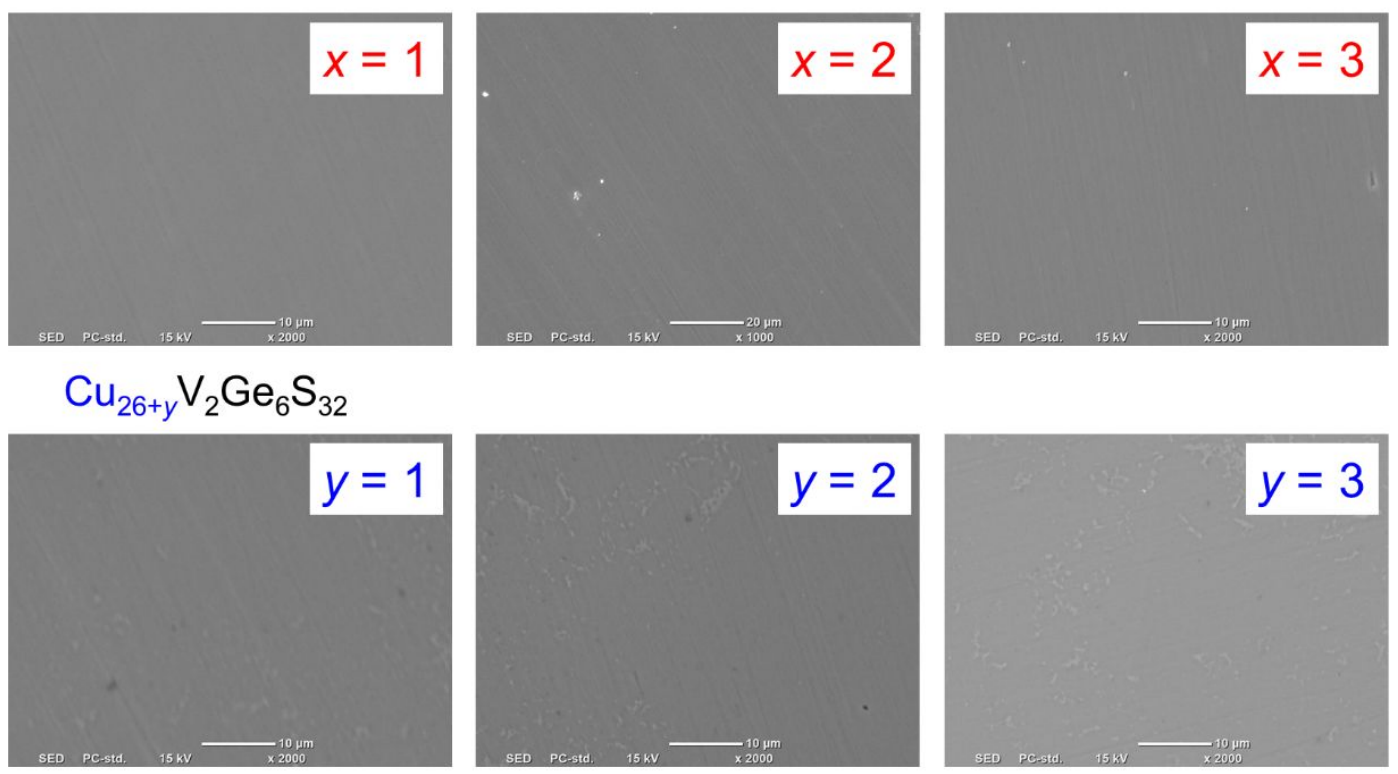

\section{$\mathrm{Cu}_{26+y} \mathrm{~V}_{2} \mathrm{Ge}_{5} \mathrm{SbS}_{32}$}
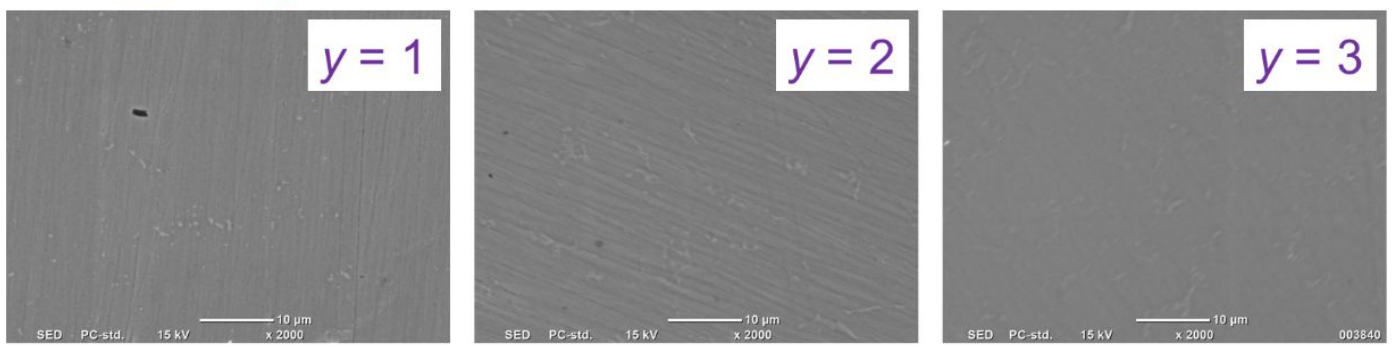

Figure S15. Scanning electron microscopy images for $\mathrm{Cu}_{26} \mathrm{~V}_{2} \mathrm{Ge}_{6-x} \mathrm{Sb}_{x} \mathrm{~S}_{32}, \quad \mathrm{Cu}_{26+y} \mathrm{~V}_{2} \mathrm{Ge}_{6} \mathrm{~S}_{32}$, and $\mathrm{Cu}_{26+y} \mathrm{~V}_{2} \mathrm{Ge}_{6-x} \mathrm{Sb}_{x} \mathrm{~S}_{32}$ with $x=1$ samples. 
Table S4. Hole carrier concentration $n$ and Hall mobility $\mu_{\mathrm{H}}$ for $\mathrm{Cu}_{26} \mathrm{~V}_{2} \mathrm{Ge}_{6-x} \mathrm{Sb}_{x} \mathrm{~S}_{32}, \mathrm{Cu}_{26+y} \mathrm{~V}_{2} \mathrm{Ge}_{6} \mathrm{~S}_{32}$, and $\mathrm{Cu}_{26+y} \mathrm{~V}_{2} \mathrm{Ge}_{6-x} \mathrm{Sb}_{x} \mathrm{~S}_{32}$ with $x=1$ samples.

\begin{tabular}{lll}
\hline & $n / 10^{21} \mathrm{~cm}^{-3}$ & $\mu_{\mathrm{H}} / \mathrm{cm}^{2} \mathrm{~V}^{-1} \mathrm{~s}^{-1}$ \\
\hline$x=y=0$ & 6.8 & 5.5 \\
$x=1, y=0$ & 5.5 & 4.0 \\
$x=2, y=0$ & 4.0 & 2.8 \\
$x=3, y=0$ & 1.9 & 1.9 \\
$x=0, y=2$ & 5.1 & 2.6 \\
$x=0, y=3$ & 4.1 & 1.6 \\
$x=1, y=3$ & 1.7 & 1.0 \\
\hline
\end{tabular}



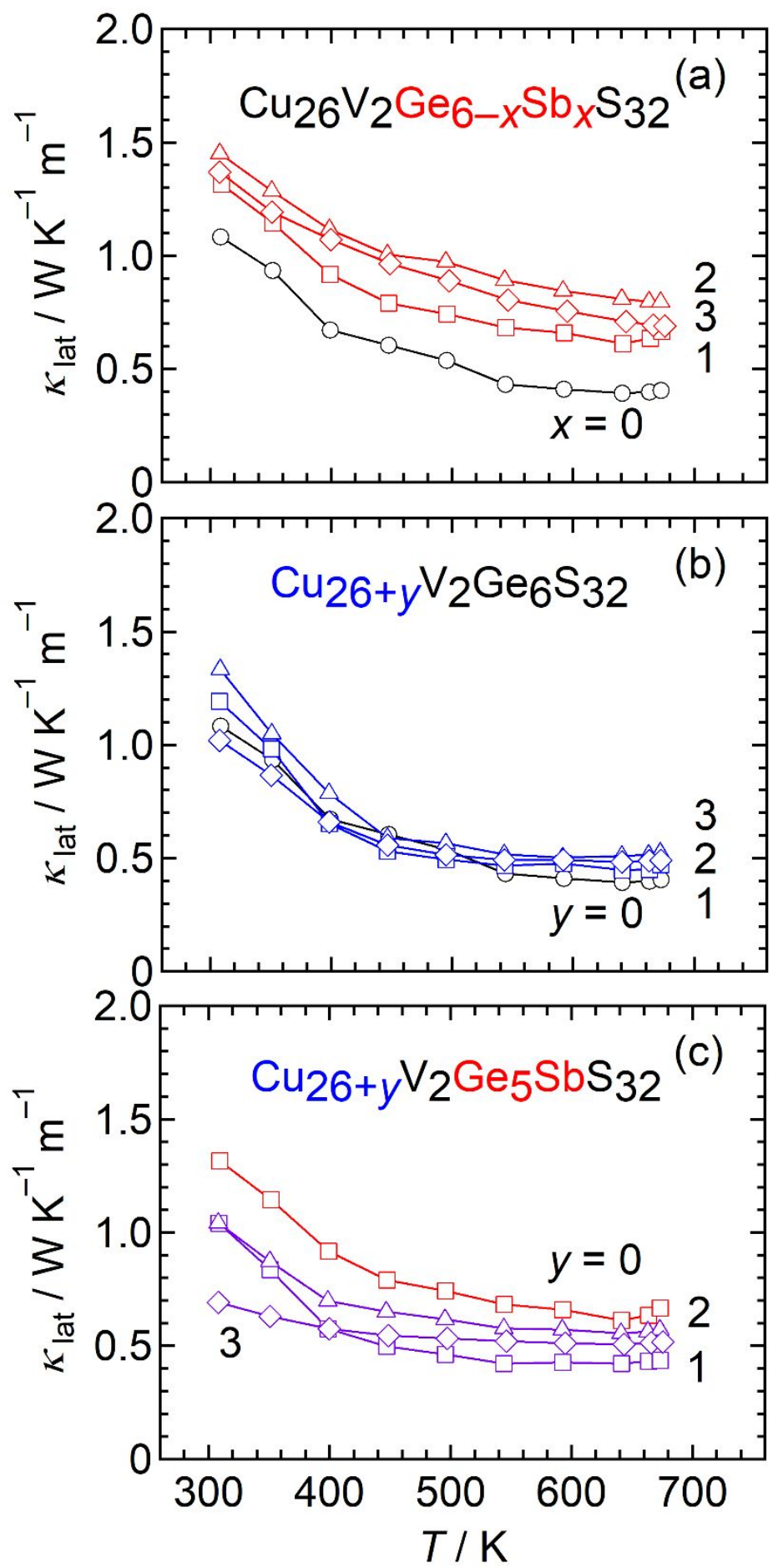

Figure S16. Lattice thermal conductivity $\kappa_{\text {lat }}$ for $\mathrm{Cu}_{26} \mathrm{~V}_{2} \mathrm{Ge}_{6-x} \mathrm{Sb}_{x} \mathrm{~S}_{32}, \mathrm{Cu}_{26+y} \mathrm{~V}_{2} \mathrm{Ge}_{6} \mathrm{~S}_{32}$, and $\mathrm{Cu}_{26+y} \mathrm{~V}_{2} \mathrm{Ge}_{6-x} \mathrm{Sb}_{x} \mathrm{~S}_{32}$ with $x=1$ samples. 